\title{
A Single Intravesical Instillation of VAX014 Inhibits Orthotopic Superficial Bladder Tumor Implantation to Increase Survival
}

\author{
BRYAN M. HANCOCK ${ }^{1}$, KATHLEEN L. MCGUIRE ${ }^{1}$, SHINGO TSUJI ${ }^{2}$, KATHERINE REIL ${ }^{2}$, \\ VERONICA HERNANDEZ ${ }^{2}$, MATTHEW J. GIACALONE ${ }^{2}$ and W. T. GODBEY ${ }^{3}$ \\ ${ }^{1}$ Department of Cell \& Molecular Biology, San Diego State University, San Diego, CA, U.S.A.; \\ ${ }^{2}$ Vaxiion Therapeutics, San Diego, CA, U.S.A.; \\ ${ }^{3}$ Department of Chemical and Biological Engineering, Tulane University, New Orleans, LA, U.S.A.
}

\begin{abstract}
Background/Aim: VAX014 minicells (VAX014) have been previously characterized as an integrin-specific oncolytic biotherapeutic agent. The present study was designed to evaluate the potential of VAX014 as an immediate post-operative intravesical adjuvant therapy in the treatment of non-muscle invasive bladder cancer (NMIBC). Materials and Methods: The ability of VAX014 to kill a panel of dissociated urothelial carcinoma cell lines was tested in vitro. In vivo experiments were conducted using a single intravesical dose of VAX014 in the antiimplantation variation of the MB49 syngeneic orthotopic bladder cancer model with tumor implantation and overall survival rates serving as study endpoints. Results: VAX014 rapidly killed dissociated urothelial carcinoma cells, while single dose in vivo pharmacology studies demonstrated the dosedependent ability of VAXO14 to prevent tumor implantation and development, ultimately resulting in a significant survival advantage compared to controls. Conclusion: These results suggest that VAX014 holds potential as an immediate postoperative adjuvant therapy in NMIBC.
\end{abstract}

Bladder cancer ranks second amongst newly diagnosed urothelial carcinomas worldwide, is the sixth leading cause of cancer death and the fourth most common malignancy of men in developed countries (1). Current estimates suggest that as many as $75-80 \%$ of patients with bladder cancer present for the first time with localized non-muscle invasive bladder cancer (NMIBC). It is further estimated that of those newly diagnosed NMIBC cases, $70 \%$ present

This article is freely accessible online.

Correspondence to: W.T. Godbey, Department of Chemical and Biological Engineering, 6823 St. Charles Ave., 300 Lindy Boggs Center, New Orleans, LA 70118, U.S.A. E-mail: Godbey@Tulane.edu

Key Words: Non-muscle invasive bladder cancer, minicells, invasin, perfringolysin $\mathrm{O}$. with what the European Association of Urologists classifies as low-risk disease (2).

Current recommended clinical practice for the treatment of low-risk NMIBC is cystoscopic transurethral resection of bladder tumor (TURBT) followed by an immediate postoperative intravesical instillation of a chemotherapeutic agent. Without adjuvant treatment, as many as $35.8 \%$ of patients treated for low-risk NMIBC will suffer recurrence within 2 years (3). There have been several studies and meta-analyses published indicating that the immediate postoperative instillation of adjuvant therapy may lead to a decrease in the overall risk of recurrence of up to $39 \%$, with absolute reduction in recurrence rates as high as $17 \%$ (4). While clearly effective in reducing recurrence, several recently conducted surveys have revealed that immediate single-dose post-operative adjuvant therapy is not consistently administered $(5,6)$. A prominently cited reason among those clinicians who do not administer immediate post-operative chemotherapy is that the potential for systemic exposure and agent-associated toxicity outweighs the clinical benefit, especially in cases where bladder perforation is suspected. The perceived clinical benefit-torisk ratio argument seems justified given that while patients with low- and intermediate-risk NMIBC have a high rate of recurrence, the initial risk of progression is low $(0.8 \%$ and $6 \%$ at 5 years, respectively) (2). However, the risk of progression increases with the number and interval of recurrences, so any initial prevention or delay of recurrence would be of clear benefit (2). Moreover, because of the high rate of recurrence, frequent monitoring and treatment are required, making NMIBC one of the most expensive cancer types to treat over the course of the disease (7). Therefore, an effective agent for use in the immediate post-operative setting would impart considerable pharmacoeconomic benefit. Recent failures in mid- and late-stage clinical trials highlight the unmet clinical need for the development of new agents that can be safely administered in the immediate post-operative setting (8). 
Different lines of evidence suggest that there are several factors lending to recurrence. These include post-TURBT reseeding of tumor cells to the urothelium, the presence of small tumors not detected during cystoscopic examination (or TURBT), and incomplete resection (9). In order to mitigate recurrence attributable to incomplete resection, many clinicians perform a second TURBT anywhere from 2-6 weeks following primary resection. While this surgical practice has helped to reduce recurrence rates, it has not eliminated recurrence altogether (10). Therefore, it is widely maintained that there remain other biological factors at play with the most likely explanation being the re-seeding of tumor cells to the urothelium. Re-seeding may be facilitated, in part, by the interaction of tumor cell integrins with extracellular matrix (ECM) components including fibronectin, laminin, and collagen, all of which are likely to be exposed at the surgical site following resection. Previous reports have indicated that specific mammalian integrins, particularly integrin heterodimer family members alpha3beta1 $(\alpha 3 \beta 1)$ and alpha5beta1 ( $\alpha 5 \beta 1)$, are implicated as being required for the attachment of tumor cells to the ECM and establishment of tumors in the urothelium in vivo (11-13).

In the present study, VAX014, a novel bacterial minicell-based product being developed for clinical investigation in multiple NMIBC subpopulations, was evaluated for its ability to prevent tumor implantation and confer a survival advantage using the 'anti-implantation' variation of the syngeneic, orthotopic MB49 murine model of bladder cancer. The anti-implantation variation of the MB49 model serves as a surrogate for the immediate postoperative treatment of NMIBC and can be useful in the pharmacological assessment of agents being investigated for this purpose $(11,14)$. Characterized in an earlier report under the research name VAX-IP, VAX014 recombinant Escherichia coli bacterial minicells (VAX014 hereafter) have been engineered to express invasin from Yersinia pseudotuberculosis to selectively target and deliver a novel bacterial protein toxin, perfringolysin $\mathrm{O}$ (PFO), to human and murine urothelial carcinoma cells (UCCs) expressing $\alpha 3 \beta 1$ or $\alpha 5 \beta 1$ (15). The mechanism of action of VAX014 is rapid permeabilization of target cell plasma membranes and simultaneous induction of apoptosis. Building upon previous in vivo studies, where repeat treatments with VAX014 conferred increased survival of animals bearing wellestablished orthotopic MB49 bladder tumors, this report demonstrates that a single intravesical dose of VAX014 is capable of preventing tumor implantation to significantly increase survival rates, making a strong argument for its clinical evaluation in the immediate post-operative setting.

\section{Materials and Methods}

Bacterial strains and minicell generation. VAX014 (invasin-positive, pfopositive), VAX-P (invasin-negative, pfo-positive) and VAX-I (invasinpositive, pfo-negative) minicells were generated from recombinant minicell-producing E. coli strains as previously described (15).
Cells. UCC lines used in this study were the murine MB49 (a kind gift from Anthony Atala, Wake Forest University Baptist Medical Center), human RT4 and human HTB-9 cell lines. Unless indicated otherwise, UCCs were maintained in Dulbecco's modified Eagle's medium (DMEM) with $10 \%$ heat inactivated fetal bovine serum (FBS), plus streptomycin and penicillin, at $37^{\circ} \mathrm{C}$ with $5 \% \mathrm{CO}_{2}$. RT4 and HTB-9 were purchased from the American Tissue Type Collection (Manassas, VA, USA).

In vitro cell-killing of murine and human UCC lines with VAX014. RT4, HTB-9 and MB49 cells were grown to $29 \%$ confluence in $75 \mathrm{~mm}^{3}$ cell culture flasks. Cells were washed once in $10 \mathrm{ml}$ sterile phosphate-buffered saline, and incubated in $1 \mathrm{ml}$ TryplE ${ }^{\mathrm{TM}}$ Express cell dissociation reagent (Life Technologies, Carlsbad, CA, USA) at $37^{\circ} \mathrm{C}$ for up to $5 \mathrm{~min}$. Following sufficient dissociation, the trypsin was inhibited by the addition of $10 \mathrm{ml}$ of fresh DMEM containing $10 \%(\mathrm{v} / \mathrm{v})$ heat-inactivated FBS, complete with penicillin and streptomycin. Cell densities were adjusted to $50,000 / \mathrm{ml}$ and $100 \mu \mathrm{l}$ were added to each well of a 96-well polystyrene tissue culture dish. For experiments with adherent cells, the cells were allowed to attach to the plate for $4 \mathrm{~h}$ before a 24-h exposure to minicells over a range of concentrations. For experiments with dissociated cells, $100 \mu \mathrm{l}$ of minicells were added immediately following the transfer of suspended cells to the 96-well plate (over a range of concentrations) and allowed to co-incubate for $2 \mathrm{~h}$. Following the allotted co-incubation time, the media were replaced, and $10 \mu \mathrm{l}$ of PrestoBlue ${ }^{\circledR}$ (Life Technologies) cell viability reagent were added. The cells were incubated for $4 \mathrm{~h}$ before plates were analyzed at Ex $560 \mathrm{~nm} / \mathrm{Em} 600 \mathrm{~nm}$.

Animals. Female C57BL/6 mice, 6-8 weeks of age (Jackson Laboratories, Bar Harbor, ME, USA), were used in all experiments and housed with normal bedding and chow with water ad libitum for the duration of the study under an animal protocol (APF\#12-11$027 \mathrm{Mc}$ ) approved by the Institutional Animal Care and Use Committee at San Diego State University.

Anti-implantation and survival experiments. The establishment of syngeneic orthotopic tumors in the MB49 murine bladder cancer model was performed as previously described using four mice per group for anti-implantation experiments and 8-10 mice per group for survival experiments $(15,16)$. After creation of urothelial burn injuries in all animals, $10^{5} \mathrm{MB} 49$ cells (in $50 \mu \mathrm{l}$ of DMEM with $10 \%$ FBS) were instilled via transurethral catheterization into the bladders of anesthetized mice and catheters kept in place for $60 \mathrm{~min}$ to prevent voiding. Bladders were then emptied and within $30 \mathrm{~min}$, mice were treated intravesically with either VAX014 minicells or saline as a negative control ( $50 \mu \mathrm{l}$ volumes). Treatment dwell times were held constant at $1 \mathrm{~h}$ after which catheters were removed and animals allowed to recover.

Statistics. Bladder weights were analyzed using the Mann-Whitney $U$-test. Survival curves were analyzed using the log-rank test.

\section{Results}

VAX014 maintains pharmacological activity following admixture with dissociated UCC lines in vitro. As a first step in evaluating the potential for clinical investigation in the immediate post-operative setting in patients with NMIBC, the ability of VAX014 to kill dissociated UCC lines (murine 

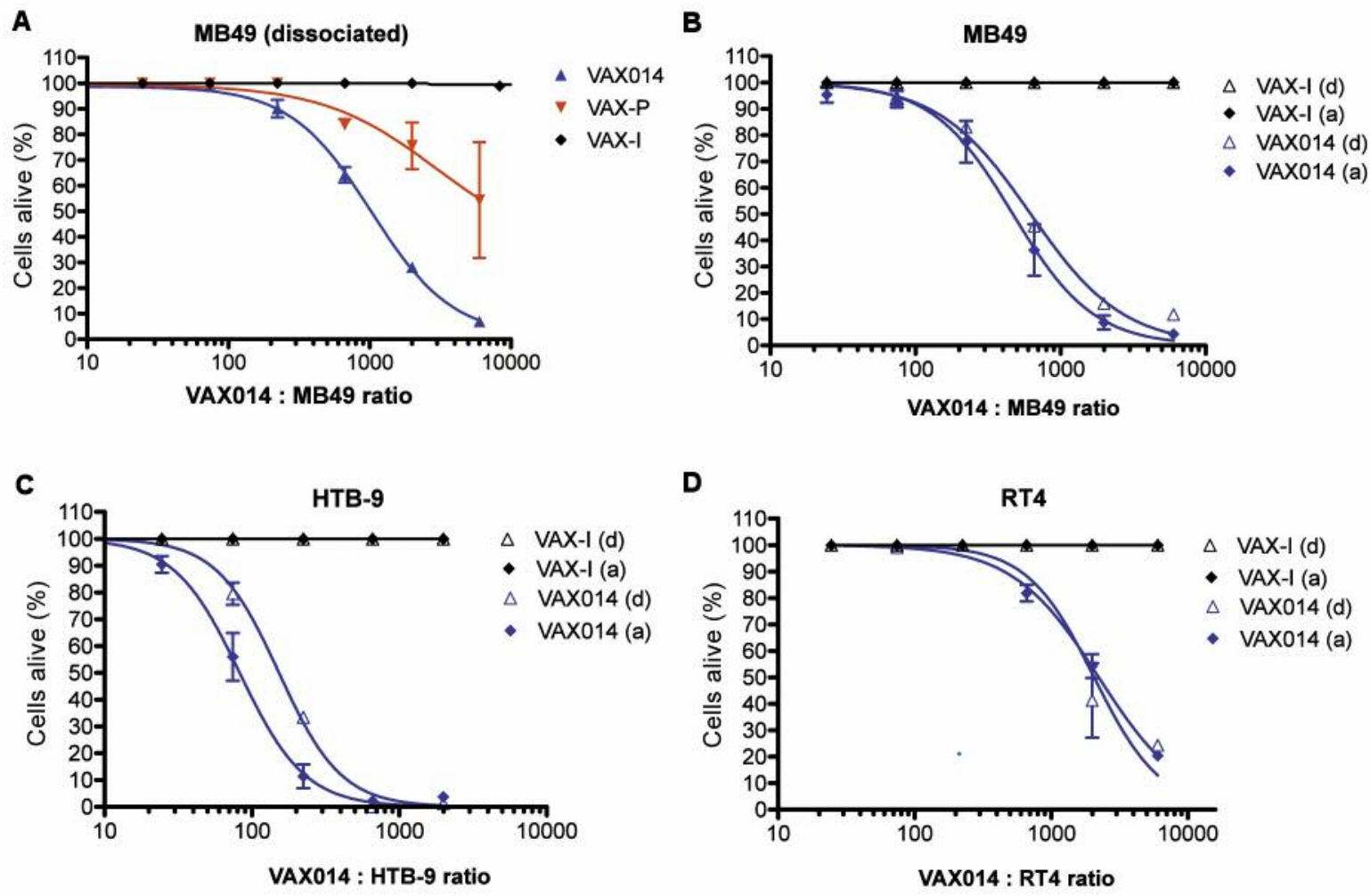

Figure 1. VAX014 is capable of rapidly killing a panel of dissociated urothelial carcinoma cell lines (UCCs) in vitro. A: VAX014, VAX-I (invasinpositive, perfringolysin O (pfo)-negative), or VAX-P (invasin-negative, pfo-positive) minicells were added to 5,000 dissociated murine MB49 UCCs at increasing ratios of minicells to MB49 cells. Cells were limited to an exposure time of $2 \mathrm{~h}$ prior to replacement of media followed by further incubation to allow for residual surviving cell growth up to $24 \mathrm{~h}$ prior to the assessment of viability using a cell proliferation assay as described in the Materials and Methods. B-D: VAX014 minicell potency against an equal number of dissociated (d) versus adherent (a) UCCs was compared after a 2-h exposure of each to VAX014. The panel of UCCs tested were murine MB49 (B), human HTB-9 (C), and human RT4 (D) cells.

MB49, human RT4 and human HTB-9) was assessed in vitro. It was reasoned that if recurrence following TURBT is due in-part to the re-seeding of tumor cells dissociated from the primary tumor during resection, then VAX014 must show sufficient pharmacological activity against dissociated UCCs. In addition, the action of VAX014 would have to be achieved with only a 2-h exposure time, as this is typically the maximum dwell time for intravesically administered agents in the clinical setting.

Taking these two aspects into consideration, it was first demonstrated that VAX014 is able to kill dissociated murine MB49 cells (Figure 1A), as compared to the control minicell types VAX-P (invasin-negative, pfo-positive) and VAX-I (invasin-positive, pfo-negative), after a 2-h exposure time. Interestingly, VAX-P minicells were somewhat effective at killing dissociated MB49 cells, albeit with a potency level at least 10-fold less than that of VAX014. The ability of VAX$P$ minicells to kill MB49 cells may be attributable to the fact that MB49 cells harbor an activating G12D mutation in the Kirsten rat sarcoma oncogene $(K R A S)$ (17). Activating mutations in both HRAS and KRAS have been shown to activate the p21-activated kinase 1 (PAK1)-dependent pathway of macropinocytosis in urothelial carcinomas, which could lead to constitutive low level uptake of untargeted minicells (18).

In follow-on experiments, the comparative potency of VAX014 against dissociated cells versus that of adherent cells was addressed. Each UCC cell line tested following a 2-h admixture of VAX014 with dissociated cells was killed with a half-maximal concentration $\left(\mathrm{EC}_{50}\right)$ at or near the $\mathrm{EC}_{50}$ value obtained when the same number of adherent cells was treated for 24-h (Figure 1B-D), whereas the control minicell type VAX-I had no effect at any of the concentrations tested. Having determined that VAX014 maintains its potency against dissociated cells as compared to adherent cells in vitro, evaluating the in vivo pharmacological activity of a single dose of VAX014 utilized in the anti-implantation variation of the MB49 model was a logical next step in assessing the potential utility of this agent in the immediate post-operative setting. 

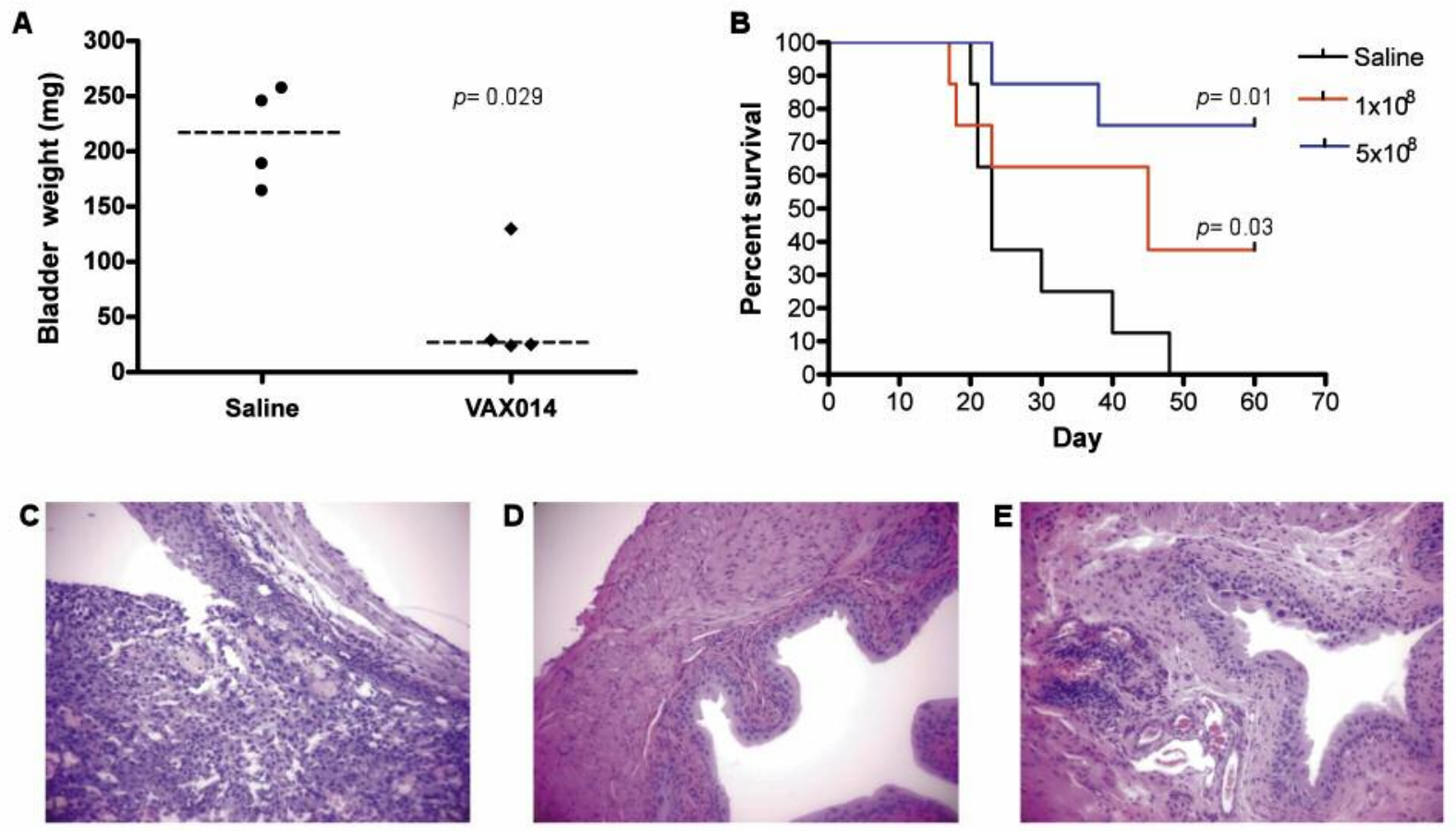

Figure 2. A single dose of VAX014 prevents tumor outgrowth and increases overall survival in the syngeneic orthotopic murine MB49 bladder cancer model. A: Female C57BL/6 mice (n=4/group) had their bladders transurethrally instilled with 100,000 MB49 cells through a urinary catheter, followed by a 1-h dwell time with a locked catheter. Immediately following the 1-h dwell time, animals were treated with $50 \mu l$ of saline or a $50 \mu l$ suspension of $1 \times 10^{8}$ VAX014 minicells and catheters were again locked for a 1-h treatment exposure. Animals were monitored daily and on day 17 were sacrificed and their bladders removed, drained, weighed and scored as a surrogate approximation of bladder tumor burden. B: Female C57BL/6 mice ( $n=8-10 /$ group) had their bladders transurethrally instilled with $10^{5}$ MB49 cells. Following a 1-h tumor cell dwell time, a single 1-h treatment with either saline, $1 \times 10^{8}$ VAX014 minicells, or $5 \times 10^{8}$ VAX014 minicells was administered intravesically in a $50 \mu l$ volume through a urinary catheter. Animals were observed twice weekly for the entire in-life observation period of 60 days. C-E: Histological confirmation of the presence of tumor in saline-treated animals that had succumbed to disease $(C)$ as compared to the absence of tumor in bladders of animals which had survived the 60-day observation period and had been treated with $1 \times 10^{8}(D)$ or $5 \times 10^{8}(E)$ VAX014 minicells. Histology was performed on $5 \mu M$ sections of formalin-fixed, paraffin embedded bladder tissues, each stained with hematoxylin and eosin ( $\times 40$ magnification).

A single dose of VAX014 prevents tumor implantation and significantly improves overall survival. The anti-implantation variation of the MB49 syngeneic orthotopic mouse model of bladder cancer was utilized in an attempt to best mimic clinical scenarios where administration of post-TURBT adjuvant therapy is recommended. In this variation, tumor cells were instilled into the bladders of female mice through a urinary catheter followed immediately by a single intravesical administration of minicells. Initial experiments assessed the effect of VAX014 on tumor growth and development after a single dose. Mice were treated with a single dose of VAX014 and sacrificed on day 17. Bladders were removed and drained, and bladder weights were recorded as a surrogate measure of tumor burden (Figure $2 \mathrm{~A})$. Dosages of $1 \times 10^{8}$ minicells were used based on our previous findings using multiple administrations in mice with well-established MB49 bladder tumors (15). This experiment demonstrated show that a single dose of $1 \times 10^{8}$
VAX014 significantly reduced tumor growth and development, compared to saline-treated controls: the VAX014-treated group had a median bladder weight of $27 \mathrm{mg}$ (range of 24-129 mg) versus the saline-treated group which had a median bladder weight of $217 \mathrm{mg}$ (range of $164-257 \mathrm{mg})(p=0.029)$. Given these results, no additionally expanded dose range-finding studies were conducted using bladder weight as the readout.

In a parallel set of studies, the ability of a single dose of VAX014 to prolong survival of tumor-bearing mice was conducted (Figure 2B). A significant survival advantage was conferred by treatment with VAX014 and the effect of treatment was dose-dependent. Treatment with a dose level of $5 \times 10^{8}$ VAX014 resulted in $75 \%$ survival at day 60 (median not reached, $p=0.01 \mathrm{vs}$. saline), and a dose level of $1 \times 10^{8}$ resulted in $37.5 \%$ survival (median at day $45, p=0.03$ $v s$. saline and $p=0.31 v s$. high-dose). The saline-treated control group had no survivors at day 60 (median at day 22). 
The bladders of all surviving animals were histologically confirmed to be completely healed and free of tumor (Figure 2C). No treatment-related systemic or local adverse sideeffects were observed at any time during the study.

\section{Discussion}

Immediate post-TURBT adjuvant therapy in patients with low-risk NMIBC has been demonstrated to impart significant clinical benefit with respect to recurrence in this patient population (3). While this is the recommended clinical practice for these patients, it is clear that inconsistent use of immediate post-TURBT adjuvant therapy is common, owing to clinician sentiment that toxicity risk outweighs clinical benefit (6). Given that administration of immediate post-TURBT adjuvant therapy can make such a meaningful impact on disease recurrence status in eligible patients, there is an obvious need for the development of safer, more effective agents.

The results of the present work demonstrate that VAX014, a novel bacterial minicell-based biopharmaceutical agent, is capable of preventing tumor cell implantation and growth while significantly improving overall survival following a single intravesical administration in a syngeneic, orthotopic mouse model of bladder cancer. As described herein and elsewhere, VAX014 selectively kills tumor cells expressing unligated $\alpha 3 \beta 1 / \alpha 5 \beta 1$ integrins (15). Both the $\alpha 3 \beta 1$ and $\alpha 5 \beta 1$ integrin family members may be of particular relevance in the context of re-seeding of tumor cells post-TURBT in NMIBC. In addition to the efficacy results shown here, the notion that integrins mediate UCC seeding is further supported by earlier work where $\alpha 5 \beta 1$ - specific and $\alpha 3 \beta 1$-specific ECM-blocking oligopeptides used in combination were able to effectively inhibit orthotopic MB49 tumor implantation (11) and in another more recent report where shRNA knockdown of $\alpha 3$ resulted in impaired establishment of human T24 UCCs in a subcutaneous xenograft model (13).

Coincidentally, both $\alpha 3 \beta 1$ and $\alpha 5 \beta 1$ are involved in wound healing, where each has been shown to mediate cell migration across newly generated ECM during the re-epithelization process and it stands to reason that $\alpha 3 \beta 1$ and $\alpha 5 \beta 1$ integrins are likely to play a role in wound healing of the urothelium post-TURBT (19). Therefore, it may be that the therapeutic benefit of VAX014 is also a product of pharmacological activity against the urothelium at the site of resection in vivo, possibly resulting from a VAX014-induced delay in ECM deposition and a theoretical reduction in available tumor reattachment sites therewith. The fact that tumor take rates with the MB49 model are reduced to zero without generation of tumor attachment sites via electrocauterization is another indirect line of evidence supporting interference with woundhealing sites as a potential secondary mechanism of VAX014 efficacy in vivo (20). Assuming this was the case in these studies, any pharmacological activity against the wound site must be transient in nature as all surviving animals suffered no symptoms of local toxicity owing to treatment and showed completely healed, scar tissue-free bladders upon histological examination. This potential pharmacological mechanism will be the subject of future studies. Regardless the outcome of those studies, this report clearly demonstrates the ability of VAX014 to kill dissociated and adherent UCCs in vitro, and to prevent tumor implantation and growth in vivo. Taken together, these findings may translate very well into the clinic as VAX014 may have equivalent pharmacodynamic activities against both the re-seeding of TURBT-dissociated tumor cells and eradication of additional small established tumors undetected during cystoscopy or TURBT, each a mechanism postulated to explain tumor recurrence in this patient population (9).

\section{Conclusion}

Significant promise of VAX014 has been demonstrated in pre-clinical efficacy studies designed to evaluate its potential for use in immediate post-operative adjuvant treatment of NMIBC in low- and intermediate-risk patient populations. These pharmacological efficacy results, in combination with the apparent safety profile of VAX014, help to support a case for future clinical investigation of this novel agent.

\section{Acknowledgements}

This work was funded by the National Institutes of Health, SBIR Phase 1 grant: 1R43CA180403-01A1.

\section{References}

1 Jemal A, Bray F, Center MM, Ferlay J, Ward E and Forman D: Global cancer statistics. CA Cancer J Clin 61(2): 69-90, 2011.

2 Babjuk M, Oosterlinck W, Sylvester R, Kaasinen E, Bohle A, Palou-Redorta $\mathrm{J}$ and Roupret M: EAU Guidelines on Nonmuscle-Invasive Uroethelial Carcinoma of the Bladder, the 2011 Update. Actas Urol Esp 36(7): 389-402, 2012.

3 Sylvester RJ, Oosterlinck W and van der Meijden AP: A single immediate postoperative instillation of chemotherapy decreases the risk of recurrence in patients with stage Ta T1 bladder cancer: A meta-analysis of published results of randomized clinical trials. J Urol 171(6 Pt 1): 2186-2190, 2004.

4 Brausi M, Witjes JA, Lamm D, Persad R, Palou J, Colombel M, Buckley R, Soloway M, Akaza $\mathrm{H}$ and Bohle A: A review of current guidelines and best practice recommendations for the management of nonmuscle-invasive bladder cancer by the international bladder cancer group. J Urol 186(6): 2158-2167,

5 Clarke NS, Basu S, Prescott S and Puri R: Chemo-prevention in superficial bladder cancer using mitomycin C: A survey of the practice patterns of British urologists. BJU Int 97(4): 716-719, 2006.

6 Cookson MS, Chang SS, Oefelein MG, Gallagher JR, Schwartz B and Heap K: National practice patterns for immediate postoperative instillation of chemotherapy in nonmuscle invasive bladder cancer. J Urol 187(5): 1571-1576, 
7 Botteman MF, Pashos CL, Redaelli A, Laskin B and Hauser R: The health economics of bladder cancer: A comprehensive review of the published literature. Pharmacoeconomics 21(18): 1315-1330, 2003.

8 Bohle A, Leyh H, Frei C, Kuhn M, Tschada R, Pottek T, Wagner W, Knispel HH, von Pokrzywnitzki W, Zorlu F, Helsberg K, Lubben B, Soldatenkova V, Stoffregen C and Buttner H: Single postoperative instillation of gemcitabine in patients with nonmuscle-invasive transitional cell carcinoma of the bladder: A randomised, double-blind, placebo-controlled phase iii multicentre study. Eur Urol 56(3): 495-503, 2009.

9 Anastasiadis A and de Reijke TM: Best practice in the treatment of nonmuscle invasive bladder cancer. Ther Adv Urol 4(1): 1332, 2012.

10 Grimm MO, Steinhoff C, Simon X, Spiegelhalder P, Ackermann $\mathrm{R}$ and Vogeli TA: Effect of routine repeat transurethral resection for superficial bladder cancer: A long-term observational study. J Urol 170(2 Pt 1): 433-437, 2003.

11 Bohle A, Jurczok A, Ardelt P, Wulf T, Ulmer AJ, Jocham D and Brandau S: Inhibition of bladder carcinoma cell adhesion by oligopeptide combinations in vitro and in vivo. J Urol 167(1): 357-363, 2002.

12 Jurczok A, Fornara P and Soling A: Bioluminescence imaging to monitor bladder cancer cell adhesion in vivo: A new approach to optimize a syngeneic, orthotopic, murine bladder cancer model. BJU Int 101(1): 120-124, 2008.

13 Li C, Yang Z, Du Y, Tang H, Chen J, Hu D and Fan Z: BCMab1, a monoclonal antibody against aberrantly glycosylated integrin alpha3beta1, has potent antitumor activity of bladder cancer in vivo. Clin Cancer Res 20(15): 4001-4013,
14 Brocks $\mathrm{CP}$, Buttner $\mathrm{H}$ and Bohle $\mathrm{A}$ : Inhibition of tumor implantation by intravesical gemcitabine in a murine model of superficial bladder cancer. J Urol 174(3): 1115-1118, 2005.

15 Tsuji S, Chen, X, Hancock B, Hernandez V, Visentin B, Reil K, Sabbadini R, Giacalone MJ and Godbey WT: Preclinical evaluation of VAX-IP, a novel bacterial minicell-based biopharmaceutical for nonmuscle invasive bladder cancer. Mol Ther Oncolytics 3: 16004, 2016.

16 Dobek GL and Godbey WT: An orthotopic model of murine bladder cancer. J Vis Exp (48): 2535, 2011.

17 Luo Y, Chen X, Han R, Chorev M, Dewolf WC and O'Donnell MA: Mutated Ras p21 as a target for cancer therapy in mouse transitional cell carcinoma. J Urol 162(4): 1519-1526, 1999.

18 Redelman-Sidi G, Iyer G, Solit DB and Glickman MS: Oncogenic activation of PAK1-dependent pathway of macropinocytosis determines BCG entry into bladder cancer cells. Cancer Res 73(3): 1156-1167, 2013.

19 Koivisto L, Heino J, Hakkinen L and Larjava H: Integrins in wound healing. Adv Wound Care 3(12): 762-783, 2014.

20 Chan E, Patel A, Heston W and Larchian W: Mouse orthotopic models for bladder cancer research. BJU Int 104(9): 1286-1291, 2009.
Received September 29, 2016

Revised October 18, 2016

Accepted October 24, 2016 